\title{
Recognizable Proof and Analysis of Palm Print in Biometric Authentication System using Bayes Techniques
}

\author{
Balaji. S, John Paul Praveen A, Mohanraj R
}

\begin{abstract}
ID and Analysis Of Palm Print In Biometric Authentication System Using Bayes TechniquesIn nowadays there is a hazard that others can get to a similar data anyplace and whenever. At present passwords, individual recognizable proof cards are utilized for individual ID. Presently a days Biometric based acknowledgment is the most prevalent human acknowledgment design. Biometrics estimates person's one of a kind or conduct attributes to validate individual personality. It gives progressively dependable and proficient methods for character check. The physical component of hand known as palm geometry contains data that is fit for confirming the personality of a person. The objective of biometrics check framework comprises in choosing whether two attributes have a place with same individual or not.In this case picture can be utilized for confirmation purposes. The term worldwide states that entire picture of the palm is considered for confirmation. Principle work for this situation is the pre-handling of picture, at that point extricating the highlights, making the informational index, bunching calculation and Classification calculation is utilized and execution is looked at in both the cases.

Index Terms - Image compression, Image recognition, Bayes Technique, Classifier.
\end{abstract}

\section{INTRODUCTION}

As of today, there is an incredible enthusiasm of numerous scientists on the Palm acknowledgment issue. Among these analysts are the designers, neuroscientists, and psychophysicists concentrating this issue in various fields and in various perspectives. There are a few application zones of Palm acknowledgment in our genuine, for example, distinguishing proof of staff utilizing charge cards, international ID checks, entrance control, criminal examinations, and so on.

Revised Manuscript Received on July 22, 2019.

Balaji .S' ECE, Bharath Institute of Higher Education and Research,India. Email: bala.sripathy@gmail.com.

John Paul Praveen, ECE, Bharath Institute of Higher Education and Research,India. Email: johnpaul.embedded@gmail.com

Mohanraj R, ECE, Bharath Institute of Higher Education and Research,India. Email: dspmohanraj@gmail.com.
Different mechanized frameworks for distinguishing proof of individuals dependent on biometrics are utilized as of

late. Alongside understood strategies, for example, unique finger impression or DNA acknowledgment, Palm acknowledgment opens new potential outcomes. Numerous requirements for putting Palm acknowledgment into training, eg, Palm confinement in advanced cameras, have just been embraced by organizations and are industrially accessible.

Palm acknowledgment is as of now being actualized into IMAGE sorting out programming, web applications , cell phones, and international IDs as of now contain Palm biometric information. This infers Palm acknowledgment is

an undeniably imperative field of biometry. The upsides of Palm acknowledgment are moderately unassuming prerequisites on equipment and straightforward continuous procedure from the perspective of the distinguished subjects.

As of late Palm acknowledgment has gotten significant consideration from scientists in biometrics, design acknowledgment, and PC vision networks. The AI and PC designs networks are additionally progressively engaged with Palm acknowledgment. This basic enthusiasm among specialists working in assorted fields is persuaded by our surprising capacity to perceive individuals and the way that human movement is an essential concern both in regular day to day existence and in the internet.

Moreover, there are countless, security, and criminological applications requiring the utilization of Palm acknowledgment advances. These applications incorporate computerized swarm observation, get to control, mugshot identification (e.g., for issuing driver licenses), Palm reproduction, plan of human PC interPalm (HCI), media correspondence (e.g., age of manufactured faces) and substance based IMAGE database the executives. Various business Palm acknowledgment frameworks have been conveyed, for example, Cognitec, Eyematic, Viisage, and Identix.

\section{II.EXISTING SYSTEM}

In multispectral imaging makes it conceivable to create powerful palm print acknowledgment techniques by using highlights acquired with various phantom wavelengths, for example, unmistakable, close infrared, infrared, and even single red, green, and blue 
channels. With infrared ghastly imaging, palm vein data would be caught to improve the capacity of farce location and the precision of palm print acknowledgment. Another fascinating course is to interest the ideal brightening (or lighting mix) for palm print acknowledgment by com=paring and consolidating the acknowledgment execution utilizing entire obvious ghostly range light and light at a few ordinary phantom groups. Until this point, a few information level, include level, and score-level combination techniques have been created for multispectral palm print acknowledgment .

\section{Disadvantages Of Existing System:}

- Recognition accuracy is less.

- There are two key challenges in accurately matching two palm print images. The first one is relating to the accurate representation of features which is seriously influenced by the noise introduced on the surface due to sweat, dirt, etc.

- The other challenge is resulting from inaccurate alignment of matched palm prints which is mainly contributed from the palm print deformations due to surface pressure such as stretching, as palm is not a rigid surface.

\section{III.MATERIALS AND METHODOLOGY}

The hardware setup plays a vital role in the designing We are proposing a system to analyze the low quality biometric recognition like palm and enhance it using bilateral filter technique and fuzzy c means clustering segmentation for palm identification. A HUMAN PALM image is taken or the acquisition can either be done via impression or direct scanning. After getting the impression converted into grey scale image to increase the properties of impression and once it converts having some noise or pixel losses that can be removed by using filters like bilateral filter. After the processing of noise removal each and every properties can be segmented using fuzzy c means clustering. Various features are extracted from the segmented image and are fed to classifiers such as Support Vector Machine. This classifier used to analyse the palm prints,Sensitivity, specificity values are analyzed to find the accuracy of the model.

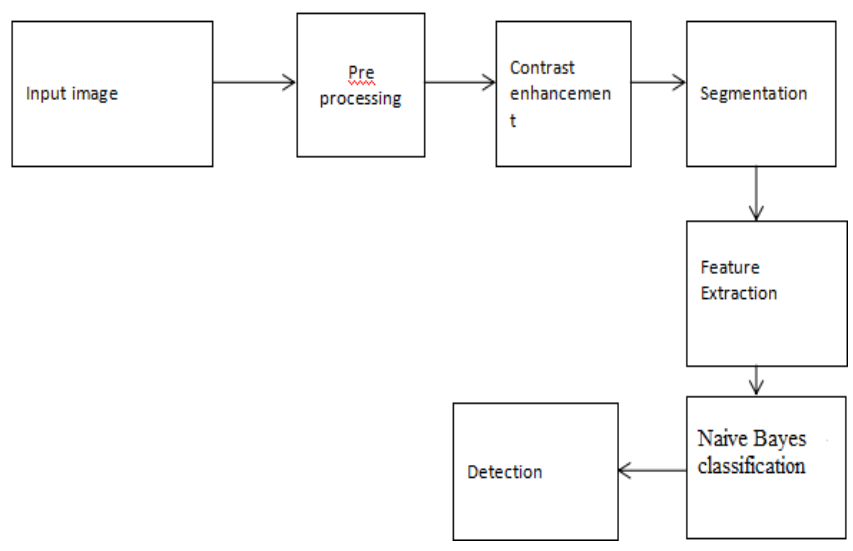

Fig 1: Block Diagram

\section{NAVE BAYES ALGORITHM DESCRIPTION}

A Naive Bayes classifier is a direct probabilistic classifier dependent on applying Bayes' hypothesis (from Bayesian estimations) with solid (gullible) self-administration suppositions. An intelligently obvious term for the covered likelihood model would be "oneself overseeing segment model". In basic terms, a legitimate Bayes classifier foresees that the closeness (or nonattendance) of a specific segment of a class is unessential to the vicinity (or nonappearance) of some other fragment. For instance, a trademark thing might be viewed as an apple if it is red, round, and around 4" in estimation. Despite whether these highlights rely on one another or upon the proximity of different highlights, a direct Bayes classifier considers these properties to autonomously add to the likelihood that this normal thing is an apple. Subordinate upon the exact idea of the likelihood model, innocent Bayes classifiers can be organized all around productively in a supervised getting the setting. In different useful applications, parameter estimation for guileless Bayes models utilizes the strategy for most unbelievable probability; continuously end, one can work with the guiltless Bayes model without believing in Bayesian likelihood or utilizing any Bayesian philosophies. Despite their guiltless game plan and over-revamped suppositions, basic Bayes classifiers have worked very well in different incredible genuine conditions. In 2004, the examination of the Bayesian game-plan issue has demonstrated that there are some theoretical explanations behind the senseless sensibility of clueless Bayes classifiers.[1] Still, a wide association with other solicitation frameworks in 2006 showed that Baye's social event is beaten by dynamically current strategies, for example, maintained trees or sporadic timberlands.

\section{V.RESULTS AND DISCUSSION}

\section{A.Image acquisition:}

Image acquisition is basically creation of database to feed into the system for training as well as for testing and classification of images. We acquired our images online from various sources. 


\section{B.Image pre-processing:}

For preprocessing the pictures are changed over into $256 \times 256$ pixels and which is later on changed into CIElab shading space. The Lab shading space depicts scientifically all discernible hues in the three measurements L for gentility and an and b for the shading adversary's green-red and blue-yellow.

\section{C.Image Segmentation:}

Picture division is the segment of a picture into a lot of non-covering areas whose association is the whole picture. In the least difficult case, one would just have an article locale and a foundation area.

A locale can't be announced a portion except if it is totally encompassed by edge pixels. It's anything but a simple undertaking to make it known to a PC what attributes establishes a "significant" division. Consequently, a lot of guidelines when all is said in done division techniques is required:

- Region insides ought to be basic and without numerous openings.

- Adjacent districts of a division ought to have fundamentally differing qualities concerning the trademark on which they are uniform.

- Boundaries of each portion ought to be basic, not worn out, and should be spatially precise.

\section{D.Feature Extraction:}

Feature extraction is done after the preprocessing stage in character affirmation system. The fundamental endeavor of model affirmation is to take a data model and precisely dole out it as one of the possible yield classes. This technique can be divided into two general stages: Feature decision and Classification. Feature assurance is essential to the whole method since the classifier won't most likely see from insufficiently picked features. Criteria to pick features given by Lippman are: "Features should contain information required to perceive classes, be harsh toward insignificant variance in the information, and moreover be limited in number, to permit, viable figuring of discriminant limits and to control the proportion of planning data required" Feature extraction is a noteworthy development in the improvement of any model portrayal and goes for the extraction of the pertinent information that depicts each class. In this system relevant features are removed from things/letters so as to edge incorporate vectors. These part vectors are then used by classifiers to see the data unit with target yield unit. It winds up less complex for the classifier to arrange between different classes by looking features as it allows really easy to perceive. Feature extraction is the technique to recoup the most huge data from the unrefined data. Feature extraction is finding the course of action of parameter that describe the condition of a character accurately and surprisingly. In feature extraction arrange, each character is addressed by a component vector, which transforms into its character.

\section{VI.CONCEPT OF IMAGE PROCESSING}

Picture arranging is a methodology to change over an Image into the front line structure and play out explicit assignments on it, to get a refreshed Image or to separate some pleasing data from it. It is a sort of standard understanding where information is Image, similar to video edge or photo and yield might be Image or attributes related to that Image. All things considered, the Image Processing framework solidifies considering Images to be two-dimensional signs while applying feasibly set flag dealing with methodologies to them.

It is among quickly making movements today, with its applications in different bits of business. Picture Processing shapes center asks about a region inside arranging and programming structuring orders moreover. In imaging science, Image preparing is any kind of flag managing for which the data is an image, for example, a photo or video design; the yield of Image arranging might be either an Image or a lot of qualities or parameters identified with the Image. Most Image preparing procedures join seeing the Image as a two-dimensional pennant and applying standard flag dealing with technique to it.

Picture dealing with all things considered infers modernized Image arranging, in any case, optical and direct Image managing besides are conceivable. This article is about general structures that apply to every one of them. The confirming of Images (passing on the information Image notwithstanding) is suggested as imaging.

Picture dealing with fundamentally unites the going with three stages.

- Importing the Image with an optical scanner or by front line photography.

- Analyzing and controlling the Image which breakers information weight and Image improvement and spotting structures that are not to human eyes like satellite photos.

- The output is the last stage wherein the result can be changed Image or report that depends upon Image examination.

Inspiration driving Image preparing:

The clarification behind IMAGE preparing is secluded into 5 social events. They are:

1. Visualization

2. Image honing and recreating

3. Image recovery.

4. Measurement of model

5. Image Recognition. 


\section{VII.CONCLUSION}

In design, we have suitably built up a solid and liberal PAI palm vessel biometric distinguishing structure for individual prominent proof. Showed up diversely about existing IR and ultrasound-based palm-vessel imaging methodologies, our structure gives better spatial targets and imaging criticalness.

\section{REFERENCES}

1. Y. Matsumoto et al., "Visualization of tumor-related blood vessels in human breast by photoacoustic imaging system with a hemispherical detector array," vol. 7, pp. 41970, 2017.

2. A. Kumar, "Human identification using palm-vein images," IEEE pp. 1259-1274, 2011.

3. NguyenThi Hoang Lan, "A Multibiometric Encryption Key Algorithm Using Fuzzy Vault to Protect Private Key in BioPKI Based Security System”, Vision for the Future (RIVF), pp. 1-6, Nov. 2010.

4. li Wang, "Incomplete fingerprint recognition based on feature fusion and pattern entropy", the Journal of China Universities of Posts and Telecommunications, Vol. 20, No.3, pp. 121-128, 2013.

5. Lalithamani1 and Sabrigiriraj,"Technique to Generate Face and Palm Vein-Based Fuzzy Vault for MultiBiometric Cryptosystem", Journal of Machine Graphics and Vision,Vol.23,No.1,pp.97-114,2014.

6. Ahmed Bouridane,"Palmprint and finger-knuckle-print for efficient person recognition based on Log-Gabor filter response", Journal of analog integration circuit signal processing, Vol. 69, pp. 17-27, 2011.
Showed up contrastingly concerning PAI based momentous finger impression biometric frameworks, our framework depends upon 3D structures. The augmentation of noteworthiness data makes it securer than 2D stand-out engraving mapping. Later on, different wavelengths can be utilized to check the hemoglobin oxygen submersion to further improve the liveness zone limit.

\section{AUTHORS PROFILE}

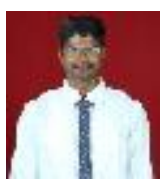

John Paul Praveen, Assistant Professor ,ECE, Bharath Institute of Higher Education and Research, Chennai,India.

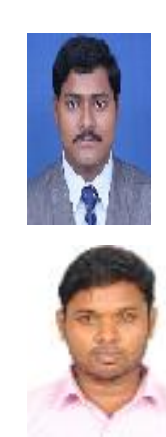

Mohanraj R, Assistant Professor, ECE, Bharath Institute of Higher Education and Research,India.

Balaji .S , Assistant Professor , ECE, Bharath Institute of Higher Education and Research,Chennai,India 